\title{
Elastic collapse in disordered isostatic networks
}

\author{
Cristian F. Moukarzel ${ }^{(a)}$ \\ Depto. de Física Aplicada, CINVESTAV del IPN, \\ Av. Tecnológico Km 6, 97310 Mérida, Yucatán, México. \\ PACS 61.43.-j - Disordered solids \\ PACS 62.20.de - Elastic moduli \\ PACS 63.50.Lm - Glasses and amorphous solids
}

\begin{abstract}
Isostatic networks are minimally rigid and therefore have, generically, nonzero elastic moduli. Regular isostatic networks have finite moduli in the limit of large sizes. However, numerical simulations show that all elastic moduli of geometrically disordered isostatic networks go to zero with system size. This holds true for positional as well as for topological disorder. In most cases, elastic moduli decrease as inverse power-laws of system size. On directed isostatic networks, however, of which the square and cubic lattices are particular cases, the decrease of the moduli is exponential with size. For these, the observed elastic weakening can be quantitatively described in terms of the multiplicative growth of stresses with system size, giving rise to bulk and shear moduli of order $e^{-b L}$. The case of sphere packings, which only accept compressive contact forces, is considered separately. It is argued that these have a finite bulk modulus because of specific correlations in contact disorder, introduced by the constraint of compressivity. We discuss why their shear modulus, nevertheless, is again zero for large sizes. A quantitative model is proposed that describes the numerically measured shear modulus, both as a function of the loading angle and system size.

In all cases, if a density $p>0$ of overconstraints is present, as when a packing is deformed by compression or when a glass is outside its isostatic composition window, all asymptotic moduli become finite. For square networks with periodic boundary conditions, these are of order $\sqrt{p}$. For directed networks, elastic moduli are of order $e^{-c / p}$, indicating the existence of an "isostatic length scale" of order $1 / p$.
\end{abstract}

Disorder in condensed matter gives rise to interesting physics that has been the object of intense study for decades 1, 2. Important problems to which much effort is still being devoted are the vibrations and electronic conduction in disordered solids [1]. Static properties like the elastic moduli of disordered systems, on the other hand, appear to be more readily accessible to analysis, so one would expect them to hold fewer surprises. This expectation turns out to be true only for hyperstatic networks. Consider, for example, a triangular spring network whose sites have been randomly displaced within a circle of radius $\epsilon$, with repose lengths of all springs adjusted accordingly. All elastic moduli of these positionally disordered $(\mathrm{PD})$ networks are finite and size-independent for large sizes, and go continuously to their homogeneous limit when $\epsilon \rightarrow 0$ 3. Formally, $Y_{L}(\epsilon) \approx Y_{\infty}-a \epsilon^{2}$ for large $L$. A similar behavior is observed for topologically disor-

(a) email address: cristian@mda.cinvestav.mx dered (TD) networks obtained e.g. by locally rewiring a small fraction $\epsilon$ of the links.

However, the simple perturbative picture described above is not universal. It is only valid for elastic networks that, like the triangular network, are Statically Indeterminate or Hyperstatic, i.e. have more springs than strictly required to be rigid. Networks which are minimally rigid and do not have redundant springs are Statically Determinate or Isostatic. Their elastic properties show, as their size increases, a dramatic disorder-weakening effect that is the subject of this work. We will argue that all elastic moduli of isostatic networks go to zero with system size, if these have random geometrical (positional or topological) disorder.

An Isostatic Network (IN) is mechanically rigid, and therefore has no flexibilities (zero-energy modes), other than the trivial $m(d)=d(d+1) / 2$ rigid roto-translations in $d$ dimensions. But the removal of any spring introduces 
a)

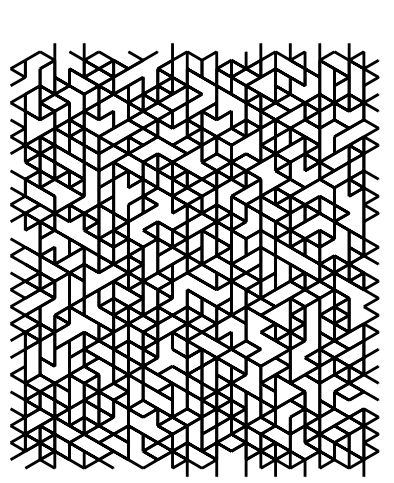

b)

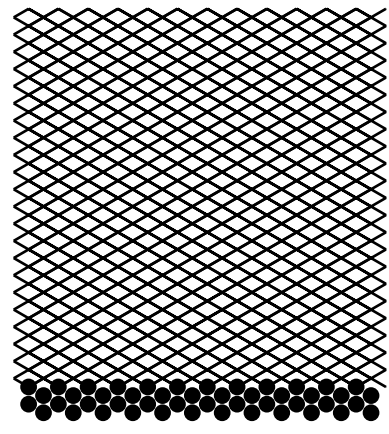

Fig. 1: a) Random isostatic network with periodic boundaries. These networks are built by means of a matching algorithm for rigidity [4. b) Positionally disordered square lattice attached to a fixed boundary (black circles) and loaded at the top, with periodic boundary condition in the horizontal direction.

one flexibility, that is, an isostatic network has no redundant springs. A simple counting of degrees of freedom $(d$ degrees of freedom per site) and constraints (each spring counts as a constraint), originally due to Maxwell [5], shows that a freely-standing isostatic network of $N$ points in $d$ dimensions has exactly $d N-m(d)$ springs. This condition is called constraint balance. Neglecting boundary contributions, their average coordination is therefore $\langle z\rangle=2 d$. It is important to remark that constraint balance is not a sufficient condition for isostaticity. Additionally, all springs must be "properly distributed", in the sense defined in the theory of graph rigidity.

While building topologically disordered isostatic networks is in general hard (satisfying constraint balance is easy, verifying proper distribution is highly nontrivial), in two dimensions simple integer algorithms exist [4, 6, for this task. On the other hand, topologically regular isostatic lattices are easily obtained. The simplest examples of such regular INs are the square and cubic lattices with appropriate boundary conditions. These are topologically ordered, as every site in the bulk has coordination $z=2 d$. Given that square and cubic lattices are so common, it is surprising that their elastic properties in the presence of positional disorder have not been discussed in detail before. Furthermore, the relevance of our findings is not limited to topologically regular isostatic networks. In recent years, it has been noticed that several natural systems self-organize onto topologically disordered structures that are isostatic, i.e. minimally rigid without redundant links. Following the discovery of isostaticity [7] in frictionless sphere-packings (which are models of metallic glasses [8], colloids 9], emulsions [10, and granular matter [7,11]), it was later proposed [12,13, that covalent glasses are isostatic in a certain composition window. These further instances of isostatic networks are topologically disordered, as the coordination number fluctuates from site to site. Isostatic systems have a

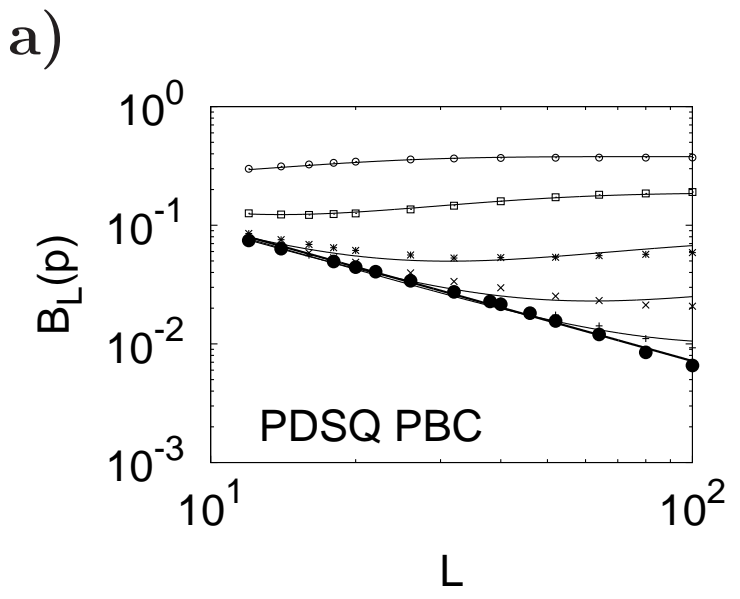

b)

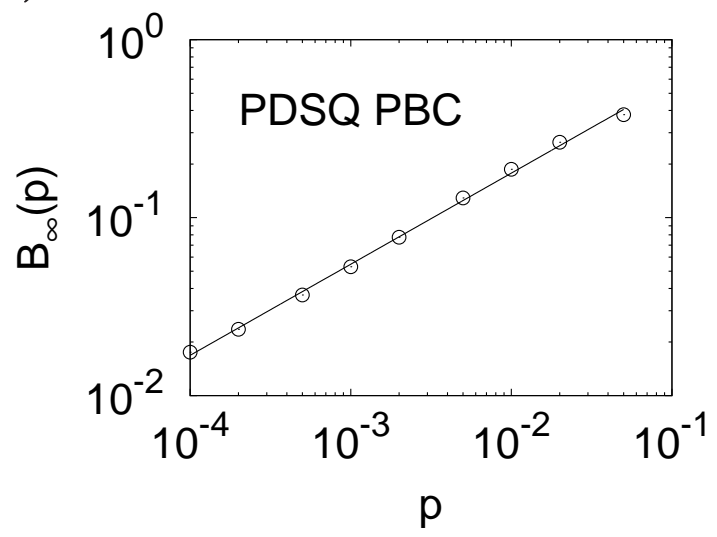

Fig. 2: a) Bulk elastic modulus $B_{L}(p)$ for PDSQ with periodic boundary conditions $(\mathrm{PBC})$ in the isostatic case (full circles) and with a density $p$ of excess springs equal to $1 \times 10^{-4}$ (plusses), $5 \times 10^{-4}$ (crosses), $2 \times 10^{-3}$ (stars), $1 \times 10^{-2}$ (squares), and $5 \times 10^{-2}$ (circles). Solid lines are fits to the data (see text). b) Asymptotic modulus $B_{\infty}(p)$ vs $p$ for PDSQ with PBC. The full line is a power law fit with exponent $\gamma=0.53 \pm 0.05$.

number of anomalous properties that derive from their being at the verge of loosing mechanical stability. Much attention has been devoted in recent years to the study of the particular static [7, 14, 19] and vibrational [18, 20 properties that isostatic systems display.

In this work, elastic properties are explored for two different types of disordered isostatic networks. These are: positionally disordered square networks (PDSQ), and topologically disordered isostatic networks (TD). The latter are built as a random subset of the triangular lattice, enforcing isostaticity by means of matching algorithm for rigidity 4. An example of TD is shown in Fig. 1. For the purpose of these studies, $\epsilon=0.2$ is taken for all disordered networks. Elastic properties of isostatic networks are strongly dependent on boundary conditions. Let us first consider two-dimensional isostatic networks with periodic boundary conditions, made of $N=L^{2}$ sites with $2 N$ (properly distributed) springs 1 .

\footnotetext{
1 Since two translations are isometries on a torus, two of these
} 
a)

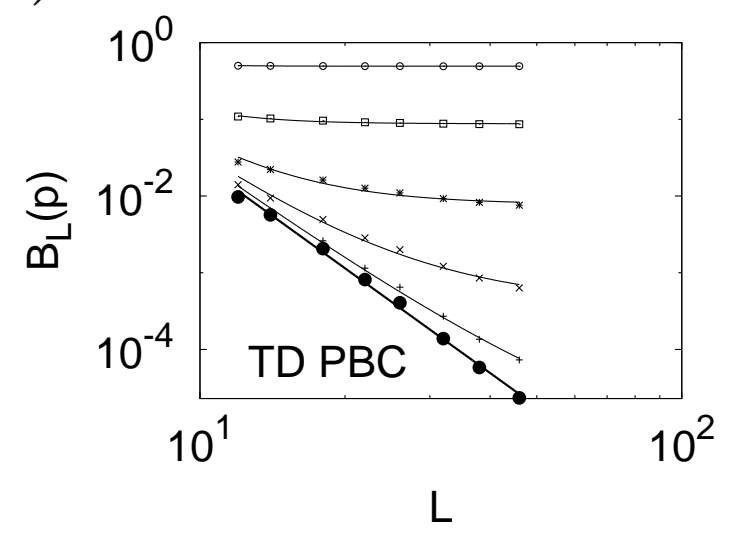

b)

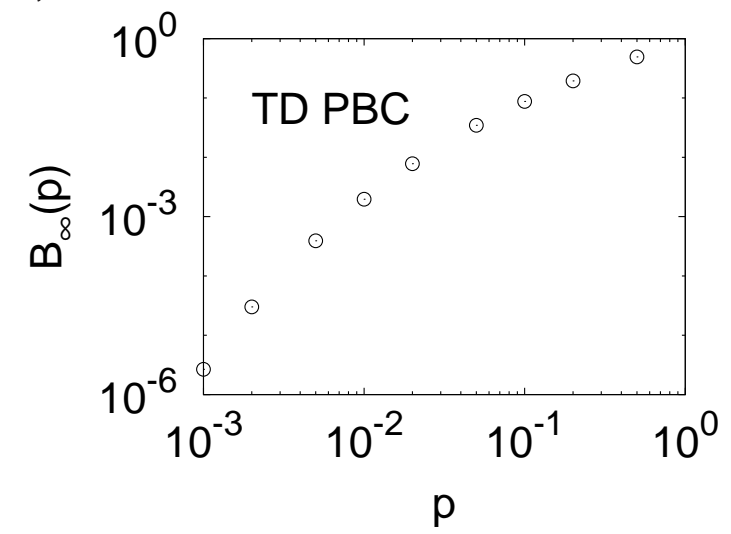

Fig. 3: a) Bulk elastic modulus $B_{L}(p)$ for TD networks with $\mathrm{PBC}$ in the isostatic case (full circles) and with a density $p$ of excess springs equal to $1 \times 10^{-3}$ (plusses), $5 \times 10^{-3}$ (crosses), $2 \times 10^{-2}$ (stars), $1 \times 10^{-1}$ (squares), and $5 \times 10^{-1}$ (circles). Solid lines are fits (see text). b) Asymptotic modulus $B_{\infty}(p)$ vs $p$ for TD networks with PBC.

The bulk elastic modulus $B$ of an elastic network with periodic boundary conditions can be measured as the per-link elastic energy due to an infinitesimal expansion of all springs. This modulus is finite and size independent for regular (topologically as well as positionally ordered) networks as e.g a regular square lattice. For PDSQ and TD networks, on the other hand, there is a disorderinduced elastic collapse, whereby $B_{L}^{\text {iso }}$ goes to zero as a power law of system size $L$, as shown in Figs. 2a and 3a. From fits to these data it is found that $B_{L}^{\text {iso }} \sim a L^{-\mu}$ with $\mu^{\mathrm{PDSQ}}=1.3 \pm 0.1$, and $\mu^{\mathrm{TD}}=5.5 \pm 0.5$. The value of $\mu$ is not universal but dependent on $\epsilon$, network structure, and boundary conditions. Furthermore, as we later show, in certain cases the decay of the modulus is not even power-law but exponential with size.

This disorder-induced elastic collapse only happens for

springs are redundant. Therefore these networks are not strictly isostatic. However, their number of redundancies is not extensive but small, and can be ignored for the purpose of our discussion. We will refer to them as being isostatic. isostatic networks. If a small density $p>0$ of extra springs per site is now added 21], these networks become overconstrained (or hyperstatic) with average coordination $z=4+2 p$. Overconstrained disordered networks have, in the large-size limit (LSL), a finite bulk modulus $B_{\infty}(p)$ (See Figs. $2 \mathrm{~b}$ and $3 \mathrm{~b}$ ). In order to estimate $B_{\infty}(p)$ for asymptotically large sizes, we fit the bulk modulus data for $p>0$ using ad-hoc expressions, since at present there is no theoretical prediction for the functional form of finite-size effects. For PDSQ networks with $p>0$ we use $B_{L}(p)=B_{L}^{\text {iso }} e^{-c(p) L}+B_{\infty}(p)\left(1-e^{-c(p) L}\right)$, with $c(p)$ and $B_{\infty}(p)$ as fitting parameters for each $p$. From the resulting fits we find that $c(p) \sim b p^{\sigma}$ with $\sigma \approx 0.5$. This behavior would suggest the existence of a crossover length of order $p^{-1 / 2}$. We notice that a characteristic length-scale in this problem is the average distance between overconstrained links, and that this length behaves precisely as $p^{-1 / 2}$ in two dimensions. The asymptotic modulus for PDSQ is found to behave as $B_{\infty}(p) \sim d p^{\gamma}$, with $\gamma=0.53 \pm 0.05$ (Fig. 2b).

For TD networks, a simple expression $B_{L}(p)=\left\{\left(B_{L}^{\text {iso }}\right)^{e}+\left(B_{\infty}(p)\right)^{e}\right\}^{1 / e}$, with $e(p)$ and $B_{\infty}(p)$ as fitting parameters, is found to describe the data in Fig. 3a acceptably well, and $e(p)$ turns out to be close to one in most cases. Our results for $B_{\infty}(p)$ of TD networks is shown in Fig. 3b, where it is seen that no single power-law of $p$ describes the data well.

We now consider the case of directed INs; those for which rigidity stems from a boundary, and whose structure is such that all spring forces can be obtained by local propagation of the loads using force equilibrium. We find that, while disorder-induced collapse happens for directed isostatic networks as well, its functional dependence with system size is entirely different than in the case of periodic boundaries. Two examples of disordered directed INs discussed here are the square positionally disordered directed network (PDDN - An example is shown in Fig. 1b) and the topologically disordered directed network (TDDN). TDDN are built by letting each site be supported by two springs, randomly chosen among its three lower neighbors on a triangular lattice (For details, see [7, 14 17). Both PDDN and TDDN are attached to a rigid boundary at the bottom, have periodic boundary conditions in the horizontal direction, and a free upper boundary where loads are applied.

Elastic moduli of these networks are proportional to the inverse per-spring elastic energy resulting from letting a unit load act on each site of their free upper boundary. If these loads are vertical, the uniaxial compression modulus $Y$ is obtained, while if the loads are horizontal, the shear modulus $G$ is measured. In the absence of disorder, directed square networks have finite uniaxial compression modulus $Y$ and shear modulus $G$. For PDDN and TDDN, on the other hand, both $Y$ and $G$ go to zero exponentially with size. Fig. 4a shows the uniaxial compression modulus of PDDN, while Fig. 4b 

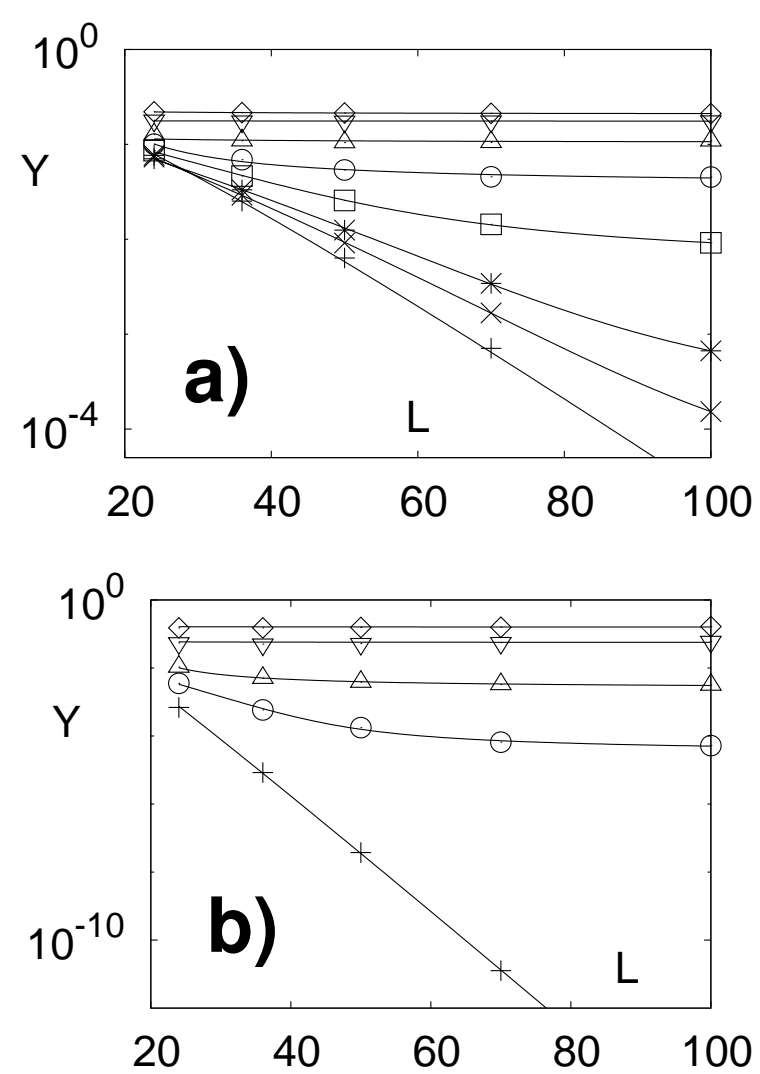

Fig. 4: Uniaxial compressive elastic modulus $Y$ for: a) PDDN with $\epsilon=0.2$, and b) TDDN, with a density of extra springs $p=0$ (isostatic case, plusses), $p=10^{-3}$ (crosses), $2 \times 10^{-3}$ (stars), $5 \times 10^{-3}$ (squares), $10^{-2}$ (circles), $2 \times 10^{-2}$ (triangles), $5 \times 10^{-2}$ (downwards triangles), and $10^{-1}$ (diamonds). Lines are fits using Eq. (1).

shows data for TDDN. The shear modulus $G$ (not shown) for PDDN and TDDN behaves in a similar way, going to zero exponentially with size.

Also in the case of directed networks, disorder-induced elastic collapse is a consequence of isostaticity. Adding a density $p>0$ of extra springs at each site, overconstrained networks are obtained. These have, in the LSL, a finite modulus $Y_{\infty}(p)$, which we estimate by fitting (the use of this expression will be justified later)

$$
Y(L, p)=b L Y_{\infty}(p) / \ln \left(\frac{a+Y_{\infty}(p) e^{b L}}{a+Y_{\infty}(p)}\right)
$$

to the data in Figs. 4a,b, with $Y_{\infty}(p), a$, and $b$ as free parameters when $p>0$. For $p \rightarrow 0$, one has $Y_{\infty} \rightarrow 0$ and (1) reduces to $Y(L)=a b L /\left(e^{b L}-1\right)$, with only two fitting parameters.

Let us now discuss these observations for directed networks. For an elastic network under a load $\sigma$ per unit area, $Y \propto \sigma^{2} / f_{2}$ 22], where $f_{2}$ is the second moment of the spring-force distribution. The vanishing of $Y$ with increasing $L$ then implies $f_{2} \rightarrow \infty$ in that limit. Fig. 5 a shows the second moment $f_{2}^{(p, L)}(d)$ of spring stresses at a fixed depth $d$ (distance from the top) for several values of $p$ and for $L=100$ and 70, on $\epsilon=0.20$ PDDNs. When $p=0, f_{2}$ grows exponentially with depth as $f_{2}^{0} e^{b d}$. For overconstrained networks with $p>0$, the growth of $f_{2}$ is initially exponential but saturates at a $p$-dependent value $f_{2}^{l i m}(p)$. The exponential growth of $f_{2}$ for $p=0$ can be understood in terms of previously reported 17, 14 17 multiplicative properties of stress propagation on directed INs. We can now provide a justification for Eq. (1). For PDDN and TDDN, since they are directed, stresses are determined propagatively from the top down, without any information at all coming from below 7, 14, 15, 17. Therefore $f_{2}^{(p, L)}(d)$ cannot depend on $L$. This is verified in Fig. 5] with $p=0$, where data for $L=100$ and 70 are seen to behave similarly. When $p>0$, propagativity no longer holds, as stresses on overconstrained networks depend on the rheology of the whole system. Despite this, Fig. 5 a shows that $f_{2}^{(p, L)}(d)$ remains approximately $L$-independent for small $p$ as well, and therefore can be approximately described by an $L$-independent expression. We choose $f_{2}(d) \sim\left(e^{-b d} / f_{2}^{0}+1 / f_{2}^{l i m}\right)^{-1}$, where $f_{2}^{l i m}(p)$ is the asymptotic value of $f_{2}$ at large depths. This simple ansatz allows us to calculate $Y^{-1}(L, p) \sim 1 / L \int_{0}^{L} d t f_{2}(t)$ from which Eq. (11) results, with $Y_{\infty} \propto 1 / f_{2}^{\text {lim }}$. As seen in Fig. 4a,b, Eq. (1) fits our data for PDDN and TDDN quite well. The resulting asymptotic values $Y_{\infty}(p)$ for PDDN and TDDN are found to be clearly incompatible with a power-law form $Y_{\infty}(p) \sim p^{\theta}$, but they can be fitted quite closely using $Y_{\infty}=y_{0} e^{-c / p}$, as shown in Fig. 5b. Writing $Y_{\infty} \sim 1 / f_{2}^{l i m} \sim e^{-b d_{s}}$, where $d_{s}(p)$ is a stress-saturation depth, this last result then implies that $d_{s} \sim 1 / p$. The following picture then emerges: on directed INs, stress fluctuations grow multiplicatively [7]14 17, their scale increases exponentially with depth, and therefore $Y_{L} \sim e^{-b L}$. For slightly overconstrained networks, this behavior is essentially unchanged, i.e. the system behaves as if isostatic, until a scale $d_{s} \sim 1 / p$ is reached, beyond which $f_{2}$ saturates and $Y_{L}$ attains a finite value $Y_{\infty}$. We notice that an 'isostaticity length' $\ell \sim 1 / p$ has been defined in the context of vibrational modes of isostatic systems [18. Our results show that this same scale determines the elastic properties of directed INs. Given that $1 / p$ also determines the average vertical separation among redundant springs, this might suggest that directed isostatic networks behave as if effectively one-dimensional, probably due to unidimensional character of stress propagation on these systems. Further work is needed to verify this picture in three dimensions.

We remark that disorder-induced elastic collapse happens for isostatic networks with any type of local structure and any sort of boundary conditions, if these have uncorrelated geometric disorder. Other forms of randomness, like mass or spring-constant disorder, which determine the dynamical properties of networks [1, do not have an elastic weakening effect on INs. Additionally, notice that appropriately chosen correlations in geometrical disorder may 

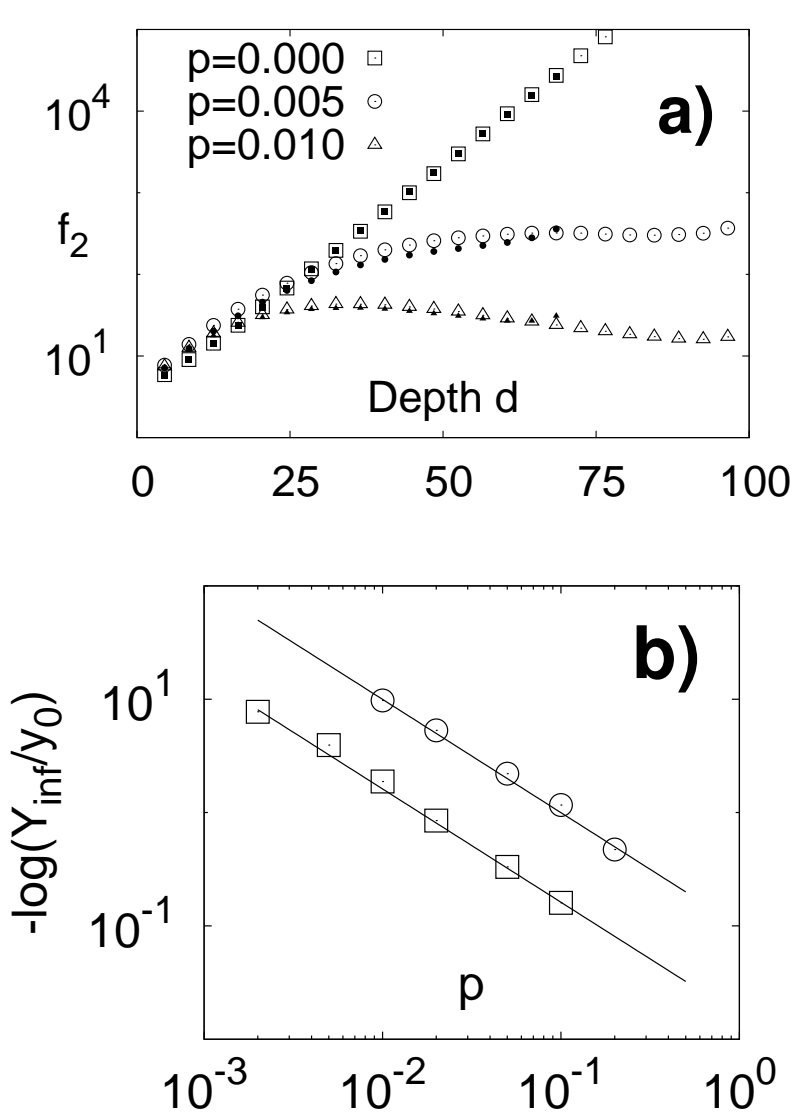

Fig. 5: a) Second moment $f_{2}$ of spring stresses at depth $d$ on PDDNs with disorder $\epsilon=0.20$, for three different values of the density $p$ of excess springs. Data shown are for sizes $L=100$ (empty symbols) and 70 (filled symbols). TDDNs show a similar behavior. b) Asymptotic compressive modulus $Y_{\infty}(p)$ versus overconstraint density $p$, for PDDN (squares) and TDDN (circles). Solid lines are fits of the form $\log Y_{\infty} / y_{0}=-c / p$.

effectively suppress elastic collapse. This is the case with compressive isostatic packings, i.e. those built under the constraint that no contact be subject to traction, as we discuss next. A system of frictionless stiff spheres self organizes to satisfy this constraint by rearranging its contacts, for a given external load (which we call the preparation load), by opening contacts subject to traction, and replacing them with compressive ones. The resulting isostatic set of contacts is disordered, but not independently so. Correlations among them have been imposed by the compressivity condition, under the given preparation load. The absence of negative forces makes large stresses impossible, as we discuss later. As a result, the elastic modulus, measured in the direction of the preparation load, is finite in the LSL. But for any load other than the preparation load (if further contact rearrangements are not allowed) the elastic response vanishes in the LSL. This has been observed in numerical studies of compressive sphere packings, where the bulk modulus is found to be finite for linear elastic interactions, but the shear modulus vanishes asymptotically, at the isostatic point 11.

The vanishing of the shear modulus while the compressive modulus is finite can be illustrated, and discussed quantitatively, with the help of a toy model for compressive sphere packings, which we call Compressive-Stress Topologically Disordered Directed Network (CSTDDN). This model is defined as follows. For a given (e.g. vertical) preparation load, contact forces are calculated by propagation from the top, layer by layer. At each site, supporting leg configurations are chosen at random, but only among the two possibilities (out of three in $2 \mathrm{~d}$ ) which ensure that all contact forces below this site are compressive (See [7, 14, 15, 17] ). This compressivity condition makes the configuration of contacts dependent upon the loads applied at the top, and also upon the particular set of contacts chosen at all intermediate steps, thereby introducing correlations. The reason why these correlated INs have finite modulus in the direction of the correlating load is simple. The sum of all vertical stress components across a given cut must equal the total load applied at the top, i.e. contact forces satisfy a "conservation equation". Since negative forces are absent by construction, contact forces cannot grow arbitrarily large with size as they do on uncorrelated isostatic networks, where large negative forces can be balanced by large positive ones so as to still satisfy this conservation rule. Therefore the scale of forces remains finite in the LSL, and so does the compressive modulus $Y$ (Fig. 6). But these correlations are ineffective to limit the scale of contact forces if now the load at the top differs in any way from the preparation load, without allowing for further contact rearrangements. Consider the case of a load that deviates by an angle $\Theta$ from the preparation load. A CSTDDN, "prepared" with a vertical homogeneous load $\sigma_{y}$, has a depth-independent second moment of stresses $f_{2}^{\sigma_{y}}=\sigma_{y}^{2} / Y_{0}$ and a finite modulus $Y_{o}$. If an additional shear $\sigma_{x}$ is now applied (without modifying the network topology), the second moment of the additional stresses will grow exponentially with depth $x$ as $f_{2}^{\sigma_{x}} \sim \sigma_{x}^{2} a e^{b x}$. By superposition, the resulting modulus $Y \sim L\left(\sigma_{x}^{2}+\sigma_{y}^{2}\right) / \int_{0}^{L}\left(f_{2}^{\sigma_{x}}+f_{2}^{\sigma_{y}}\right) d x$ is

$$
Y=Y_{0} \frac{1+\Theta^{2}}{1+a Y_{0} \Theta^{2}\left(e^{b L}-1\right) / b l},
$$

where $\Theta=\sigma_{x} / \sigma_{y}$ measures deviation of the measurement load from the direction of the preparation load. As seen in Fig. 6, our numerical data for the elastic modulus in this model is described by this expression very well. Simple analysis of (2) reveals three regimes that are clearly recognizable in Fig. 6] where $Y$ behaves respectively as i) $Y \sim Y_{0}$ for $\Theta^{2}<e^{-b L} / Y_{0}$, ii) $Y \sim \Theta^{2} e^{-b L}$ for $e^{-b L} / Y_{0}<\Theta^{2}<1$, and iii) $Y \sim e^{-b L}$ for $\Theta>1$.

We have thus shown that, while regular isostatic networks have finite elastic moduli, the elastic constants of geometrically disordered isostatic networks vanish in the limit of large system sizes, although in a manner that depends on structure and boundary conditions. INs with periodic 
boundary conditions have a bulk elastic modulus that goes to zero as a power-law of size $L$ with a non-universal exponent. For the case of directed INs grounded to a rigid bar and loaded at the opposite end, all moduli go to zero exponentially with size

The vanishing of elastic modulus with size has been pre-

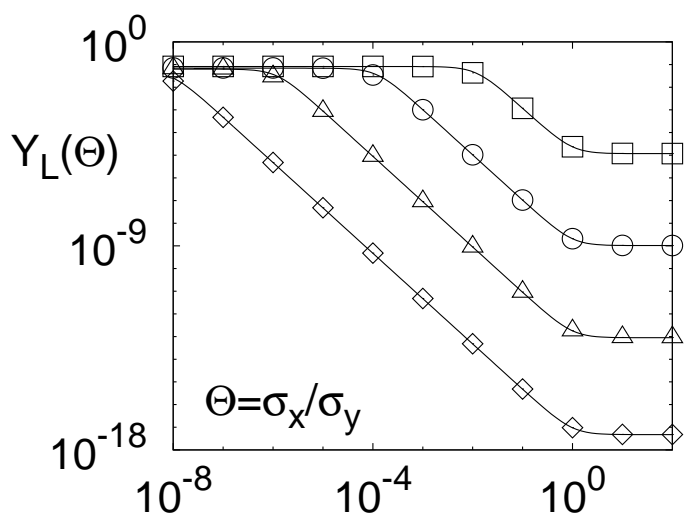

Fig. 6: Elastic modulus of CSTDDNs, a toy model for compressive sphere packings, measured at an angle $\Theta$ from the preparation load, for sizes $L=100$ (squares), 200 (circles), 300 (triangles), and 400 (diamonds). Solid lines are fits to (2).

viously reported [13] for self-organized isostatic networks. These backbones, being the isostatic subset of a random rigidity percolation backbone [23, are necessarily fractal. We have shown that even compact isostatic structures, including the square and cubic lattices, have vanishing moduli in the large-size-limit if they are geometrically disordered.

As a closing remark, we note that models of selforganization in glasses [12,24 propose that certain calcogenides may form isostatic structures in order to lower their elastic energy by avoiding self-stress. This argument should be modified, in the light of the results reported here, when a large enough external load $F=\sigma \Omega$ acts on the melt. The total elastic energy per unit volume is $e_{\text {self }}+e_{\text {load }}$ where $e_{\text {load }} \sim \sigma^{2} / Y$ is maximized for an isostatic structure, as we have shown. The minimal energy structure would then be determined by a load-dependent balance between two opposing effects: reducing self-stress by making the structure closer to isostatic, and increasing the elastic modulus by making the structure more overconstrained.

The author thanks M. Wyart, P. Duxbury and M. V. Chubynsky for useful discussions.

\section{REFERENCES}

[1] Ziman J. M., Models of Disorder 2nd Edition (Cambridge Univ. Press., Oxford) 1982.
[2] Zallen R., The Physics of Amorphous Solids (John Wiley \& Sons, New York) 1998.

[3] Mastilovic S., Int. J. Damage Mech. , 17 (2008) 357.

[4] Moukarzel, C, J. Phys. A-Math. Gen., 29 (1996) 8079.

[5] Maxwell J. C., Philos Mag ER , 27 (1864) 294.

[6] Jacobs D. and Hendrickson B., J. Comput. Phys., 137 (1997) 346.

[7] Moukarzel C. F., Phys. Rev. Lett. , 81 (1998) 1634.

[8] Wang W. H., Wang R. J., Li F. Y., Zhao D. Q. and PAN M. X., Appl. Phys. Lett. , 74 (1999) 1803.

[9] Kamp S. W. and Kilfoil M. L., Soft Matter, 5 (2009) 2438.

[10] Mason T. G., Bibette J. and Weitz D. A., Phys. Rev. Lett. , 75 (1995) 2051.

[11] O'Hern C. S., Silbert L. E., Liu A. J. and Nagel S. R., Phys. Rev. E , 68 (2003) 011306.

[12] Thorpe M., Jacobs D., Chubynsky M. and Phillips J., J. Non-Cryst. Solids, 266 (2000) 859.

[13] Chubynsky M. V. and Thorpe M. F., Curr. Opin. Solid State Mat. Sci. , 5 (2001) 525.

[14] Moukarzel C. F., Granul. Matter, 3 (2001) 41.

[15] Moukarzel C. F., J. Phys.-Condes. Matter, 14 (2002) 2379.

[16] Moukarzel C. F., Random multiplicative response functions in granular contact networks in Challenges in Granular Physics, edited by Halsey T. and Menta A., (World Scientific, Singapore) 2003.

[17] Moukarzel C. F., Response functions in isostatic packings in The Physics of Granular Media, edited by HinRICHSEN H. and Wolf D. E., (Wiley-VCH, Berlin) 2005 pp. 23-43.

[18] Wyart M., Ann. Phys.-Paris, 30 (2005) 1.

[19] Mao X. M., Xu N. and Lubensky T. C., Phys. Rev. Lett. , 104 (2010) 085504.

[20] Xu N., Wyart M., Liu A. J. and Nagel S. R., Phys. Rev. Lett. , 98 (2007) 175502.

[21] Moukarzel C. and Duxbury P., Phys. Rev. E , 59 (1999) 2614.

[22] Dearcangelis L., Redner S. and Coniglio A., Phys. Rev. B , 31 (1985) 4725.

[23] Moukarzel C. and Duxbury P., Phys. Rev. Lett. , 75 (1995) 4055.

[24] Wang Y., Boolchand P. and Micoulaut M., Europhys. Lett. , 52 (2000) 633. 